\title{
High-resolution seismic processing by Gabor deconvolution
}

\author{
Zengbao Chen ${ }^{1,2}$, Yanghua Wang ${ }^{2}$, Xiaohong $\mathrm{Chen}^{1}$ and Jingye $\mathrm{Li}^{1}$ \\ ${ }^{1}$ National Engineering Laboratory for Offshore Oil Exploration, China University of \\ Petroleum, Beijing, 102249, China. \\ ${ }^{2}$ Centre for Reservoir Geophysics, Department of Earth Science and Engineering, Imperial \\ College London, SW7 2BP, UK.
}

\begin{abstract}
Since viscoelastic attenuation effects are ubiquitous in subsurface media, the seismic source wavelet rapidly evolves as the wave travels through the subsurface. Eliminating the source wavelet and compensating the attenuation effect together may improve seismic resolution. Gabor deconvolution can achieve these two processes simultaneously, by removing the propagating wavelet which is the combination of the source wavelet and the attenuation effect. The Gabor deconvolution operator is determined based on the Gabor spectrum of a nonstationary seismic trace. By assuming white reflectivity, the Gabor amplitude spectrum can be smoothed to produce the required amplitude spectrum of the propagating wavelet. In this paper, smoothing is set as a least-squares inverse problem, and is referred to as regularized smoothing. By assuming that the source wavelet and the attenuation process are both minimum phased, the phase spectrum of the propagating wavelet can be defined by the Hilbert transform of the natural logarithm of the smoothed amplitude spectrum. The inverse of the complex spectrum of the propagating wavelet is the Gabor deconvolution operator. Applying it to the original time-frequency spectrum of the nonstationary trace produces an estimated time-frequency spectrum of reflectivity series. The final time-domain highresolution trace, obtained by an inverse Gabor transform, is close to a band-pass filtered version of the reflectivity series.
\end{abstract}

Keywords: Gabor deconvolution, inverse $Q$ filter, reflectivity inversion, high resolution.

\section{Introduction}

High-resolution seismic data are prerequisite for reservoir characterization. However, the earth's $Q$ filtering effect inevitably induces the energy dissipation of high-frequency wave components, and distorts the seismic wavelets, simultaneously (Futterman 1962, Anderson et al 1977, Kjartansson 1979, Wang and Guo 2004). Inverse $Q$ filtering and deconvolution are two common methods for seismic resolution enhancement. Typically, the former is used to compensate for viscoelastic attenuation (Hargreaves and Calvert 1991, Wang 2002, 2006, 2008), while the latter is used to eliminate the wavelet and broaden the bandwidth (Robinson 1967, Yilmaz 2001).

To remove the attenuation effect, inverse $Q$ filtering has been designed as a type of deconvolution in the plane-wave domain (Bickel and Natarajan 1985) or a type of migration 
akin to Stolt frequency-wavenumber migration (Hargreaves and Calvert 1991). A stable and efficient approach proposed byWang $(2002,2006,2008)$ is based on the theory ofwavefield downward continuation and implemented in a layered manner or in Gabor domain. Subsequently, a stationary deconvolution can be applied to remove the seismicwavelet. As an alternative, Margrave (1998) presented a nonstationary convolution model which addresses the earth's attenuation. Then Margrave and Lamoureux (2002) developed a nonstationary deconvolution using a Gabor transform. Margrave et al (2003a) presented a computational scheme for a Gabor transform based on a set of windows that form a partition of unity. The Gabor transform approximately factorizes a nonstationary trace into the product of the Fourier transform of the source wavelet, the complexvalued time-frequency attenuation function, and the forward Gabor transform of the reflectivity. The Gabor deconvolution algorithm derived from this spectral factorization attempts to estimate and remove both the source signature and the earth's attenuation effect. The latter two items are combined and is called the propagating wavelet.

The magnitude spectrum of the propagating wavelet is estimated by performing a smoothing process to the Gabor magnitude spectrum of the nonstationary seismic trace. The phase function is then calculated as in the stationary case by assuming minimum phase. When the original Gabor spectrum is divided by this time-frequency spectrum of the propagating wavelet, the result is an estimate of the Gabor spectrum of the reflectivity. An inverse Gabor transform recovers the timedomain reflectivity (Margrave et al 2011).

The Gabor deconvolution algorithm combines the essential ideas of the traditional Wiener deconvolution and inverse $Q$ filtering. Consequently, the seismic resolution is remarkably improved by the Gabor deconvolution, which corrects both wavelet shape and viscoelastic attenuation simultaneously. Ahadi and Riahi (2013) applied the Gabor deconvolution to zerooffset VSP data. The Gabor deconvolution operator was designed using the downgoing wavefield and was applied to the upgoing wavefield, and the hyperbolic smoothing was used to estimate the propagating wavelet.

Gabor deconvolution is a fully data-driven processing technology without the need of an explicit $Q$ model. In this paper, there are a number of aspects that should be highlighted in various implementation stages. First, for the Gabor transform, we use a pair of Gabor transforms considering the discrete effect (Wang 2006), which can perfectly reconstruct the original signal in numerical form. Second, for smoothing process, we investigate a regularized smoothing method as an alternative to the hyperbolic smoothing method, for the magnitude spectrum of the propagating wavelet. Finally, synthetic and field seismic data examples demonstrate that Gabor deconvolution is able to produce a seismic image with remarkably high resolution.

\section{A nonstationary trace model}

The stationary convolution model of a seismic trace, $s_{\text {stat }}(t)$, is expressed in the time domain as

$$
S_{\text {stat }}(t)=\int_{-\infty}^{\infty} w(t-\tau) r(\tau) d \tau,
$$

where $w$ is a seismic wavelet, and $r$ is a time-domain reflectivity series. Its numerical implementation may be illustrated in Figure 1: a seismic trace is a superposition of a source wavelet scaled by reflectivity samples, and the superposition process is a matrix-vector multiplication, in which the vector is the reflectivity series, and the matrix in this case is made of a stationary wavelet at different time. 


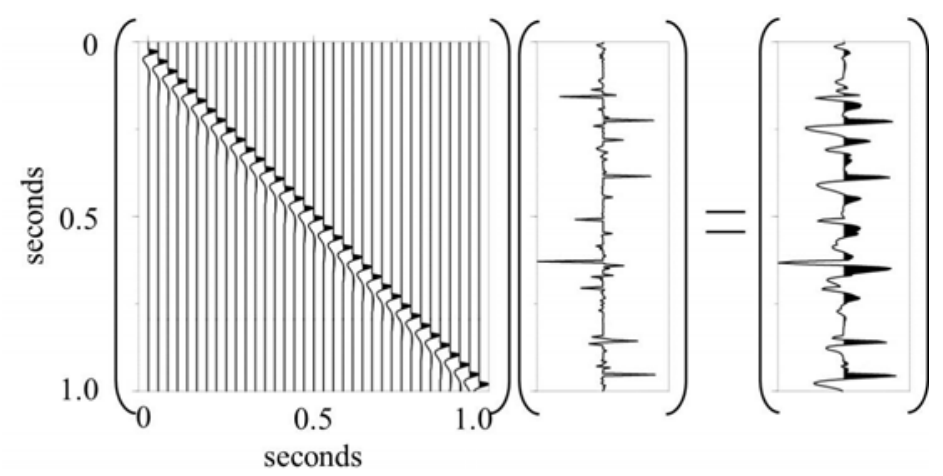

Figure 1. A depiction of stationary convolution: a Toeplitz matrix formed from a source wavelet multiplying a vector of reflectivity series. The resultant trace is a stationary superposition of a source wavelet scaled by reflectivity samples at different times.

When the "wavelet" put in the matrix is nonstationary, it is referred to as an nonstationary trace model. The nonstationary wavelet is a propagating wavelet, which includes the source signature and the nonstationary effect of dissipation described by a $Q$ model. The impulsive effect of the attenuation can be modeled by

$$
s_{Q}(t)=\int_{-\infty}^{\infty} \int_{-\infty}^{\infty} \alpha(\tau, f) r(\tau) e^{2 \pi i f(t-\tau)} d f d \tau .
$$

If denoting the inner integral over the frequency as

$$
a(\tau, t-\tau)=\int_{-\infty}^{\infty} \alpha(\tau, f) e^{2 \pi i f(t-\tau)} d f,
$$

equation (2) can be written as

$$
s_{Q}(t)=\int_{-\infty}^{\infty} a(\tau, t-\tau) r(\tau) d \tau
$$

In matrix-vector form, it is

$$
\mathbf{s}_{Q}=\mathbf{A r} .
$$

where $\mathbf{A}$ is a non-Toeplitz matrix representing $a(\tau, t-\tau)$, and $\mathbf{r}$ is a vector containing the normal-incidence reflection coefficients at two-way traveltimes. This is a nonstationary convolution model of an impulse series (Margrave, 1998).

When applying a general source signature with a stationary convolution to $\mathbf{s}_{Q}$, it generates a seismic trace, as

$$
\mathbf{s}=\mathbf{W A r},
$$

where $\mathbf{W}$ is a Toeplitz matrix formed with $w(t)$, and $\mathbf{s}$ is the vector of seismic samples. If set $\mathbf{W}_{Q}=\mathbf{W A}$ a seismic trace is the product of this nonstationary matrix $\mathbf{W}_{Q}$ with the reflectivity vector $\mathbf{r}$ :

$$
\mathbf{s}=\mathbf{W}_{Q} \mathbf{r}
$$

In the non-absorptive limit $Q \rightarrow \infty, \mathbf{A}$ may be reduced to an identity matrix and $\mathbf{W}_{Q}=\mathbf{W A}$.

The numerical computation of equation (7) for a nonstationary seismogram is depicted in Figure 2. The essential distinction between Figure 1 and Figure 2 is that the matrix in the 
latter shows a wavelet that is continually evolving according to the attenuation model, instead of an unchanging wavelet.

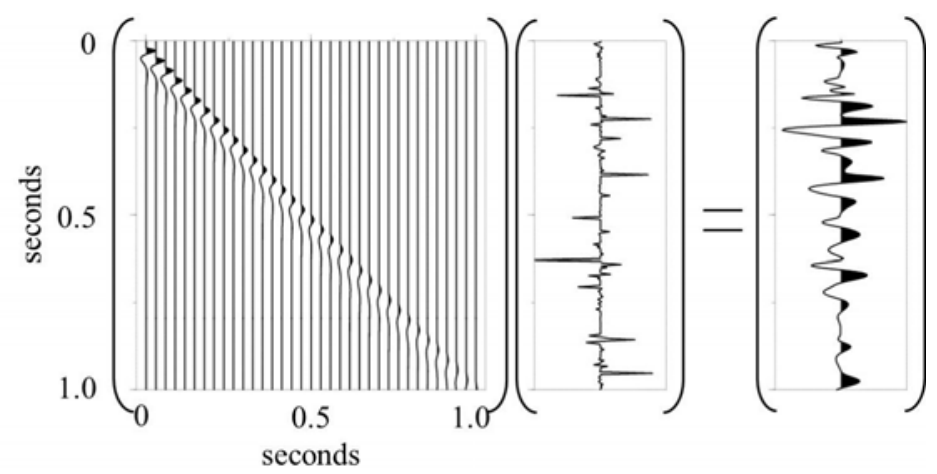

Figure 2. A depiction of nonstationary convolution. In the matrix-vector product, the matrix does not possess Toeplitz symmetry. Each column of the matrix contains the source waveform modified by the attenuation process for a traveltime equal to the column time.

The constant- $Q$ attenuation function (Kjartansson, 1979) is

$$
\alpha(\tau, f)=\exp \left(-\frac{\pi f \tau}{Q(\tau)}+i \mathrm{H}\left(-\frac{\pi f \tau}{Q(\tau)}\right)\right),
$$

where $Q$ is a variable of time $\tau$, and $\mathrm{H}$ (.) denotes the Hilbert transform over frequency $f$ at any constant $\tau$. For a layered medium, where $Q$ takes a different value in each layer, the average $Q$ is defined (Wang 2004) by

$$
\frac{1}{Q(\tau)}=\frac{1}{\tau} \sum_{k=1}^{n} \frac{\Delta \tau_{k}}{Q_{k}}
$$

where $\Delta \tau_{k}$ and $Q_{k}$ are the interval traveltime and interval $Q$, respectively, at the $k$ th layer, and $\tau$ is the total traveltime.

\section{Gabor deconvolution}

Performing Gabor transform to the nonstationary trace model (equation 6) produces Gabor spectrum as

$$
S_{g}(\tau, f) \approx W(f) A(\tau, f) R_{g}(\tau, f),
$$

where $W(f)$ is the Fourier transform of the source wavelet, $A(\tau, f)$ is the attenuation function, and $R_{g}(\tau, f)$ is the forward Gabor transform of the reflectivity series. For fixed $\tau$, since Gabor transform is just a Fourier transform (Appendix), equation (10) is a temporally localized version of the stationary convolution model. Gabor deconvolution in the Gabor (time-frequency) domain is written as

$$
R_{g}(\tau, f)=S_{g}(\tau, f) D(\tau, f),
$$

where $D(\tau, f)$ is the Gabor deconvolution operator presented in time-frequency domain. Comparing (10) and (11), we see that $D(\tau, f)=[W(f) A(\tau, f)]^{-1}$.

The operator $D(\tau, f)$ is generated as the following.

First, preforming a smoothing process over the Gabor spectrum of the nonstationary 
seismic trace to estimate the magnitude spectrum, $\left|\mathbf{W}_{Q}\right|_{\text {est }}$, of the propagating wavelet. Then, assuming both the source wavelet and the attenuation process be minimum phased, the phase spectrum $\phi(\tau, f)$ can be determined by the Hilbert transform of the natural logarithm of the amplitude spectrum over frequency $f$ at constant $\tau$ as

$$
\phi(\tau, f)=\int_{-\infty}^{\infty} \frac{\ln \left(\left|\mathbf{W}_{Q}\right|_{\text {est }}+\mu\left|\mathbf{W}_{Q}\right|_{\max }\right)}{f-f^{\prime}} d f^{\prime},
$$

where $\left|\mathbf{W}_{Q}\right|_{\max }$ is the maximum of $\left|\mathbf{W}_{Q}\right|_{\text {est }}$.

The Gabor deconvolution operator is given as (Margrave et al., 2011)

$$
D(\tau, f)=\frac{1}{\left|\mathbf{W}_{Q}\right|_{\text {est }}+\mu\left|\mathbf{W}_{Q}\right|_{\max }} e^{-i \phi(\tau, f)}
$$

where $\mu$ is a small, dimensionless stabilization constant, which is related to the noise level as well. To set a smaller value for the stabilization constant, random noise attenuation may be performed prior to Gabor deconvolution.

Finally, an inverse Gabor transform to $R_{g}(\tau, f)$ recovers the time-domain reflectivity series. For an accurate inverse Gabor transform, please refer to the Appendix.

The major steps of the Gabor deconvolution algorithm are graphically displayed in Figure 3. In the Gabor magnitude spectrum $\left|S_{g}(\tau, f)\right|$ of an attenuated trace, the progressive decay of spectral content is clearly visible (Figure 3a). Rapid fluctuation in the spectrum is due to the white reflectivity. A smoothing process to $\left|S_{g}(\tau, f)\right|$ makes an approximation to a spectrum of the propagating wavelet (Figure $3 \mathrm{~b}$ ). Finally, the estimated Gabor magnitude spectrum of the reflectivity series (Figure 3c) is comparable to the true spectrum (Figure 3d).

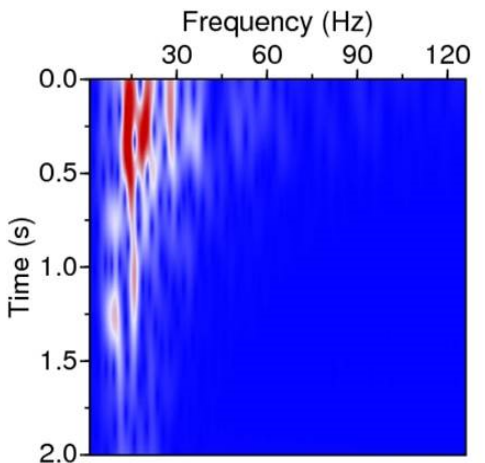

(a)

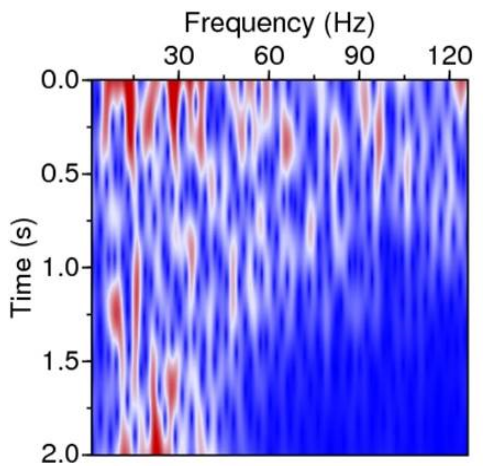

(c)

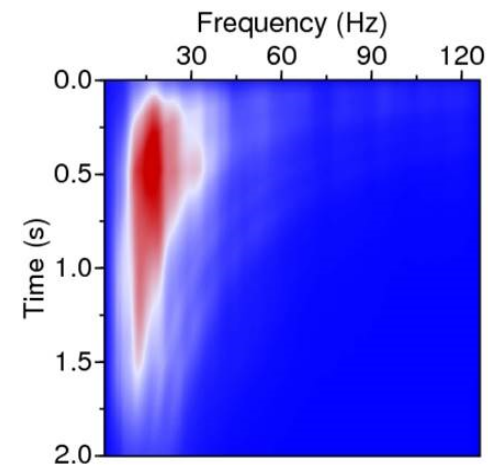

(b)

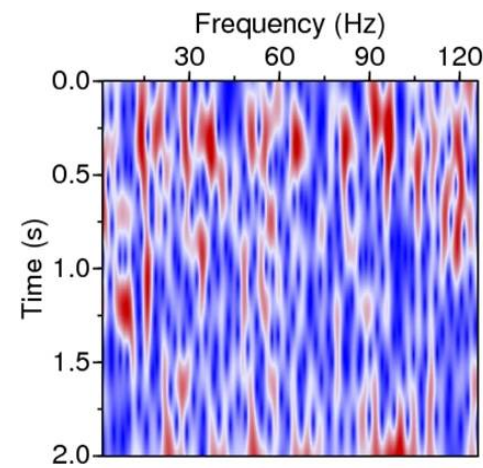

(d)

Figure 3. A depiction of the Gabor deconvolution algorithm. In each part, the image is a Gabor magnitude spectrum: (a) An attenuated seismic trace. (b) The propagating wavelet estimated by smoothing the spectrum (a). (c) Estimated spectrum of the reflectivity series and obtained from Gabor deconvolution. (d) The actual spectrum of the reflectivity series. 


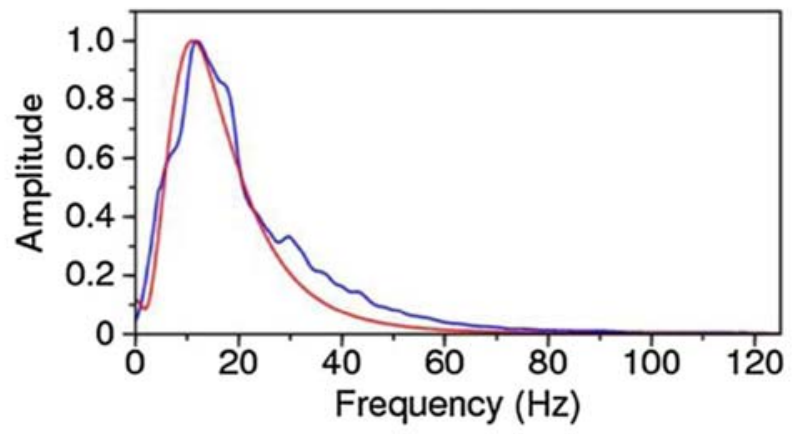

Figure 4. A comparison between the real wavelet spectrum (red curve) and the smoothed spectrum (blue curve) at time $1 \mathrm{~s}$, which is marked as a dashed yellow line in figure 3(b).

Figure 4 compares the real wavelet spectrum and the estimated wavelet spectrum at traveltime $1 \mathrm{~s}$. This comparison indicates the feasibility of estimating the propagating wavelet by smoothing the spectrum. As a field seismic trace is band-limited, Gabor deconvolution is not able to recover the low-frequency components $(<5 \mathrm{~Hz})$, as shown in figures $3(c)$ and $(d)$.

\section{Smoothing}

In the Gabor transform domain, the spectrum of the reflectivity series is much more rapidly varying than that of a propagating wavelet. Hence, the spectrum of the propagating wavelet can be estimated by smoothing over the spectrum of a seismic trace.

Hyperbolic smoothing (Iliescu and Margrave, 2002) is a robust smoothing method. The idea is originated from the constant- $Q$ operator in equation (8) that the magnitude is constant along hyperbolic curves of $\tau f=$ constant. Taking averages of the Gabor magnitude spectrum along such hyperbolic curves, the attenuation effect can be estimated (Wang, 2004). Once the attenuation is estimated, the source wavelet can be estimated subsequently by a 2-D boxcar smoothing of the spectrum after removing the attenuation (Margrave et al., 2003b, 2011).

The Gabor deconvolution operator can be determined alternatively by a regularized smoothing method. That is, smoothing is implemented as Tikhonov's regularization. The forward operator is set simply to be the identity matrix. The least-squares solution has the form (Fomel, 2007)

$$
\mathbf{m}=\left(\mathbf{I}+\varepsilon^{2} \mathbf{D}^{\mathrm{T}} \mathbf{D}\right)^{-1} \mathbf{d},
$$

where $\mathbf{d}$ is a "data" vector of time-frequency spectrum of the seismic trace, $\mathbf{D}$ is a regularization operator, and $\mathbf{m}$ denotes the required smooth "model" estimated from the data d. Smoothness is controlled by the scaling parameter $\varepsilon$, and the regularization operator $\mathbf{D}$, which can be chosen as a differential operator.

\section{Application examples}

We apply the Gabor deconvolution algorithm on both synthetic and field data to improve seismic resolution.

Figure 5 is a constant- $Q$ synthetic data example. Convolving a reflectivity series (figure 5(a)) with a wavelet generates a synthetic trace, on which a forward $Q$ filter has been applied (figure 5(b)). In this example, the wavelet is minimum-phased, with a dominant frequency of $15 \mathrm{~Hz}$, and $Q=30$ in forward $Q$ filtering. Compared with the stationary trace (figure 5(c)), the viscoelastic attenuation is more serious, as the time increases. 


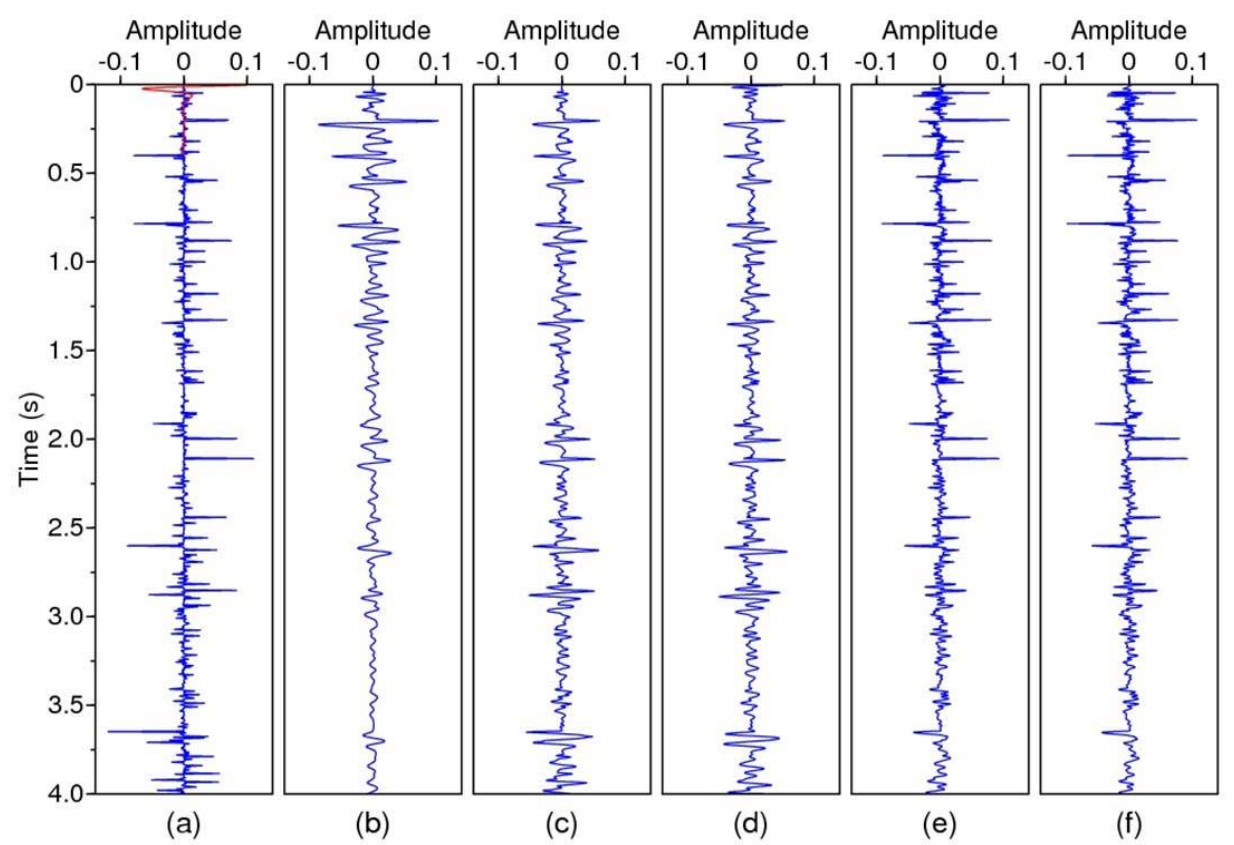

Figure 5. Constant- $Q$ synthetic data example. (a) Band-limited reflectivity and a minimum phase source wavelet (red) overlapped. (b) Nonstationary trace generated by convolving the reflectivity with the source wavelet, and then applying a forward $Q$ filter $(Q=30)$. (c) Stationary trace generated by only convolving the reflectivity with the source wavelet. (d) The result of inverse $Q$ filtering by Gabor transform. (e) The result of Gabor deconvolution using hyperbolic smoothing. ( f ) The result of Gabor deconvolution using regularized smoothing.

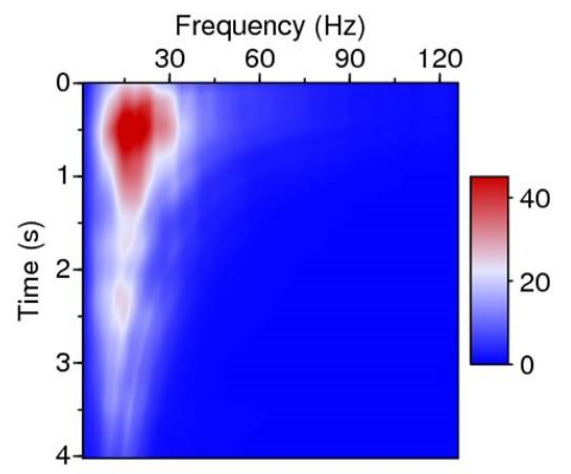

(a)

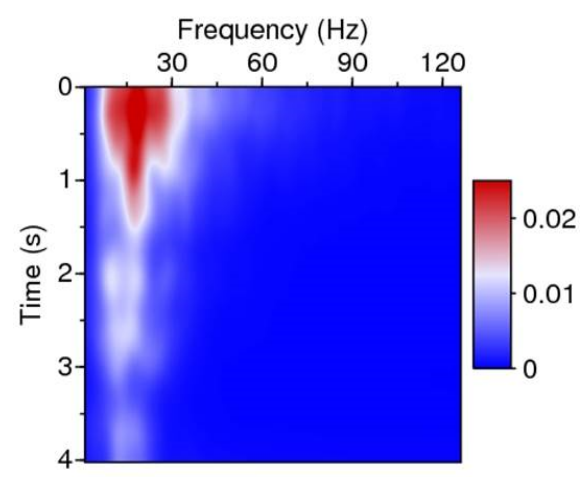

(b)

Figure 6. A comparison between the results of hyperbolic smoothing (a) and regularized smoothing (b), applied to the Gabor magnitude spectrum of the nonstationary trace shown in figure 5(b).

We perform both inverse $Q$ filtering and Gabor deconvolution to the attenuated trace. The result of inverse $Q$ filtering with the exact $Q$ value (figure $5(d)$ ) is comparable to the unattenuated trace (figure $5(c)$ ). Two results of Gabor deconvolution using hyperbolic smoothing and regularized smoothing, shown in figures $5(e)$ and $(f)$ respectively, have very little difference in between. This is because smoothed spectra of hyperbolic smoothing and regularized smoothing are comparable (figure 6). However, the result of regularized smoothing is more reasonable than that of hyperbolic smoothing, as there are no hyperbolic trails and it seems more physical in the very early time.

The displays in figures 6(a) and (b) also show different scale, corresponding to different smoothing methods. Therefore, an energy balancing between the input trace and the Gabor deconvolution result is needed. 


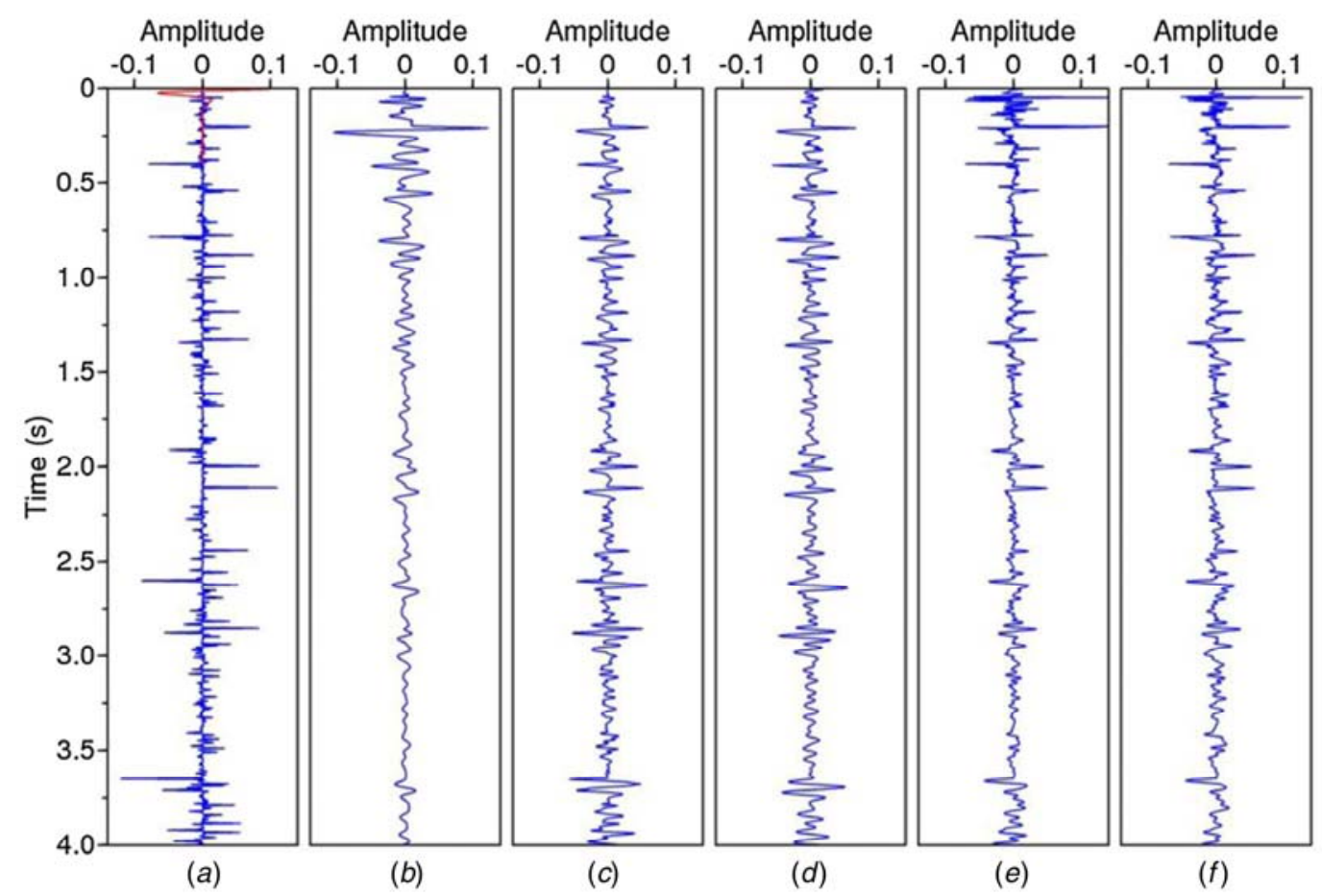

Figure 7. Variable- $Q$ synthetic data example. (a) Band-limited reflectivity and a minimum phase source wavelet (red) overlapped shown the same as in figure 5(b). (b) Nonstationary trace generated by convolving the reflectivity with the source wavelet, and then applying a forward $Q$ filter determined by the variable-Q model in table 1. (c) Stationary trace generated by only convolving the reflectivity with the source wavelet shown the same as in figures 5(c). (d) The result of inverse $Q$ filtering by Gabor transform. (e) The result of Gabor deconvolution using hyperbolic smoothing. ( f ) The result of Gabor deconvolution using regularized smoothing.

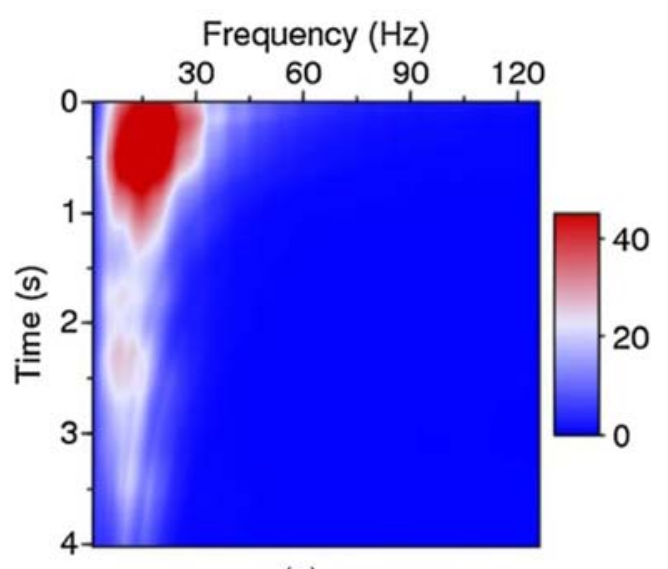

(a)

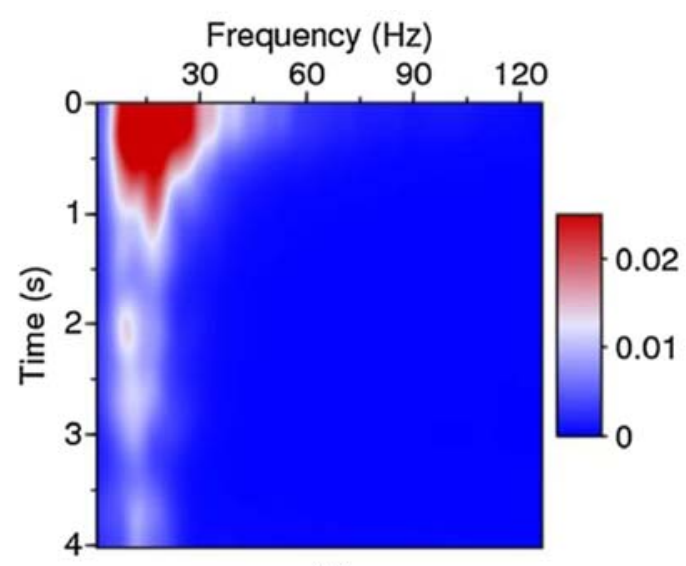

(b)

Figure 8. A comparison between the results of hyperbolic smoothing $(a)$ and regularized smoothing $(b)$, applying to the Gabor magnitude spectrum of the nonstationary trace shown in figure $7(b)$.

Figure 7 is a variable- $Q$ synthetic data example. The forward $Q$ filter is designed by a variable- $Q$ model listed in table 1 . The average- $Q$ could be continuous as its definition (equation (9)) possesses cumulative effect. We perform the same processing procedure to the attenuated trace (figure $7(b)$ ), and get similar results to the constant- $Q$ example. The result of inverse $Q$ filtering with the interval- $Q$ values (figure $7(d)$ ) only removes the attenuation. The two results of Gabor deconvolution using hyperbolic smoothing (figure 7(e)) and regularized smoothing (figure $7(f)$ ) are also comparable, due to the similar smoothed spectra (figure 8 ). 


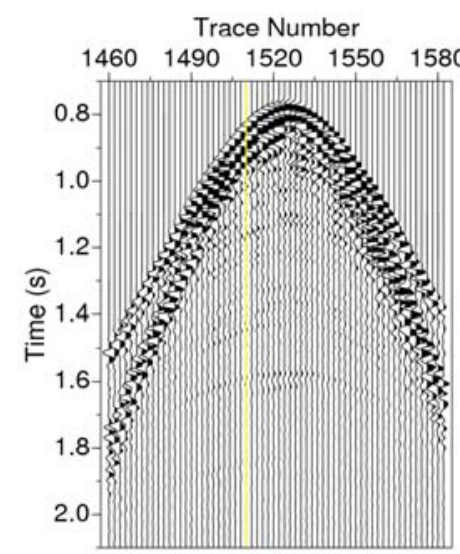

(a)

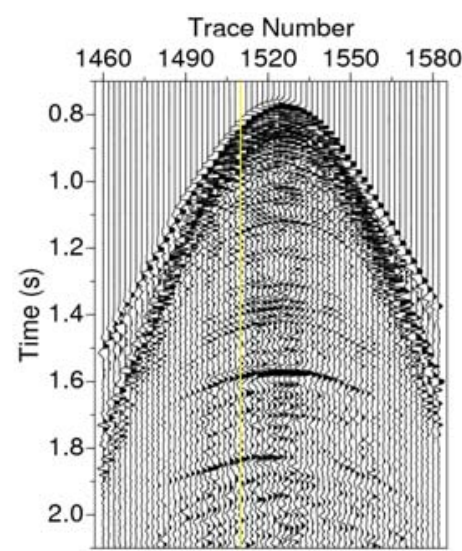

(b)

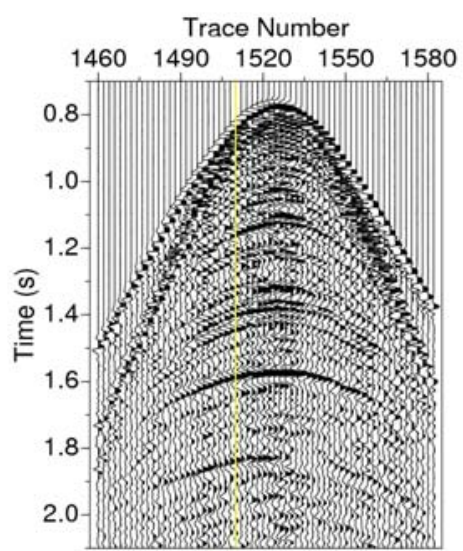

(c)

Figure 9. Gabor deconvolution applied to prestack seismic data. (a) Prestack record before Gabor deconvolution. (b) The result of Gabor deconvolution using hyperbolic smoothing. (c) The result of Gabor deconvolution using regularized smoothing. All the parameters in b and c are the same except for the smoothing method.

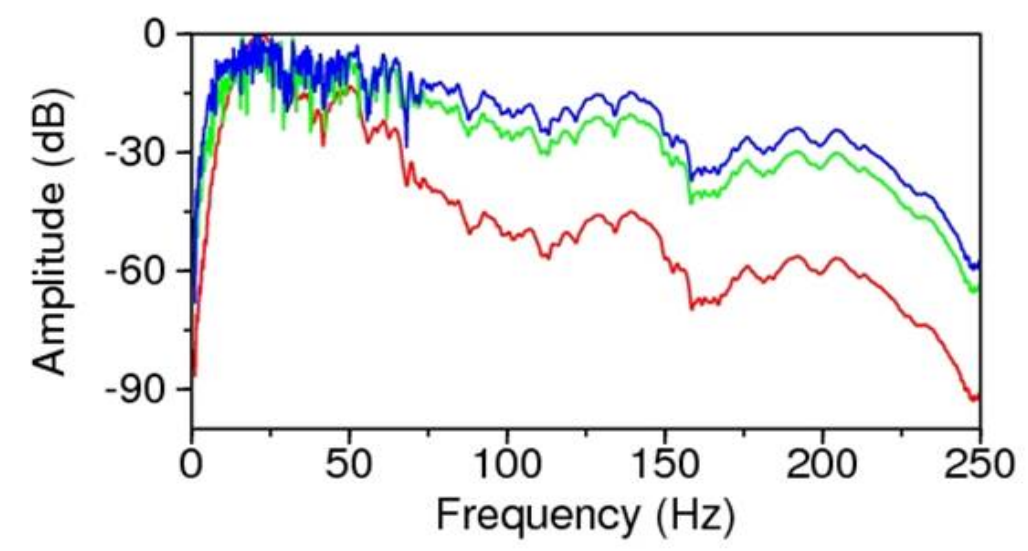

Figure 10. A comparison among the amplitude spectra of the same trace shown yellow in Figure 9 before (red) and after applying Gabor deconvolution using hyperbolic smoothing (green) and regularized smoothing (blue).

Figure $9(a)$ is a prestack field seismic record. It is original without muting the direct wave. It comes from a 3D land field data set, and the line is far from the source so that there is no surface wave. The Gabor deconvolution is applied trace by trace, therefore the lateral continuity of the events illustrate that the method is obviously effective, especially for the deep time, and it adapts to the large amplitude change immediately following the direct arrival. If comparing figures $9(b)$ and $(c)$, it is found that using the regularized smoothing method one can get a little higher resolution than hyperbolic smoothing.

Figure 10 compares the amplitude spectra of the same trace marked by yellow line in figure 9. It confirms that the spectrum of a trace after Gabor deconvolution becomes reasonably white.

We also compare the results of Gabor deconvolution using the two different smoothing methods in poststack seismic data. Figure $11(a)$ is a migration section with a high signal-tonoise ratio. After applying Gabor deconvolution trace by trace, a desirable seismic image with high resolution (figures $11(b)$ and $(c)$ ) is produced. Figures $12(a)$, $(b)$ and $(c)$ are the zoomedin sections of the rectangular regions in figure 11, respectively. The fault in this area is preserved and the events in the ovals are improved. 


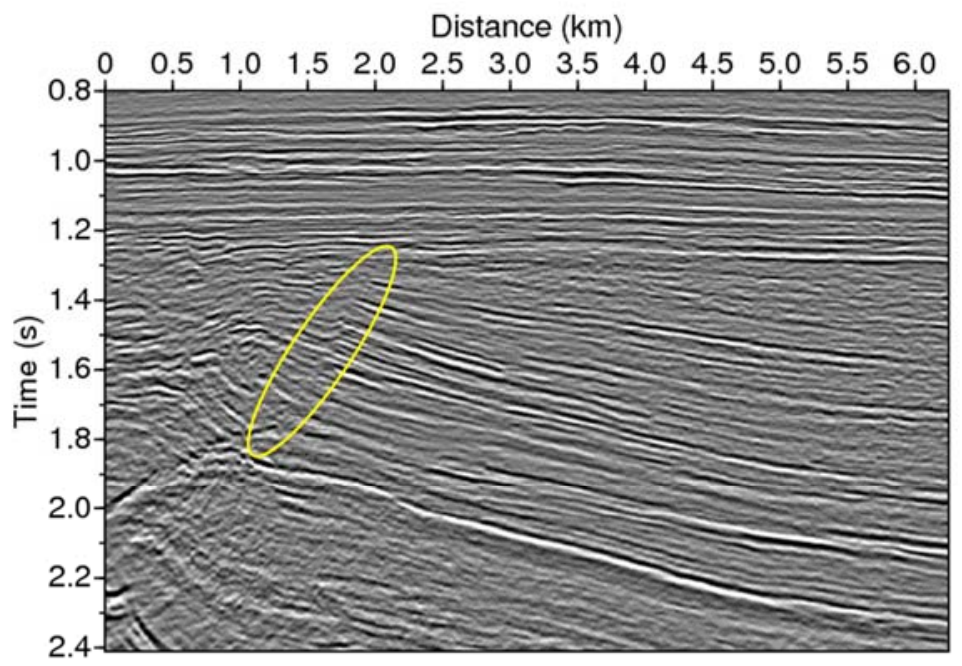

(a)

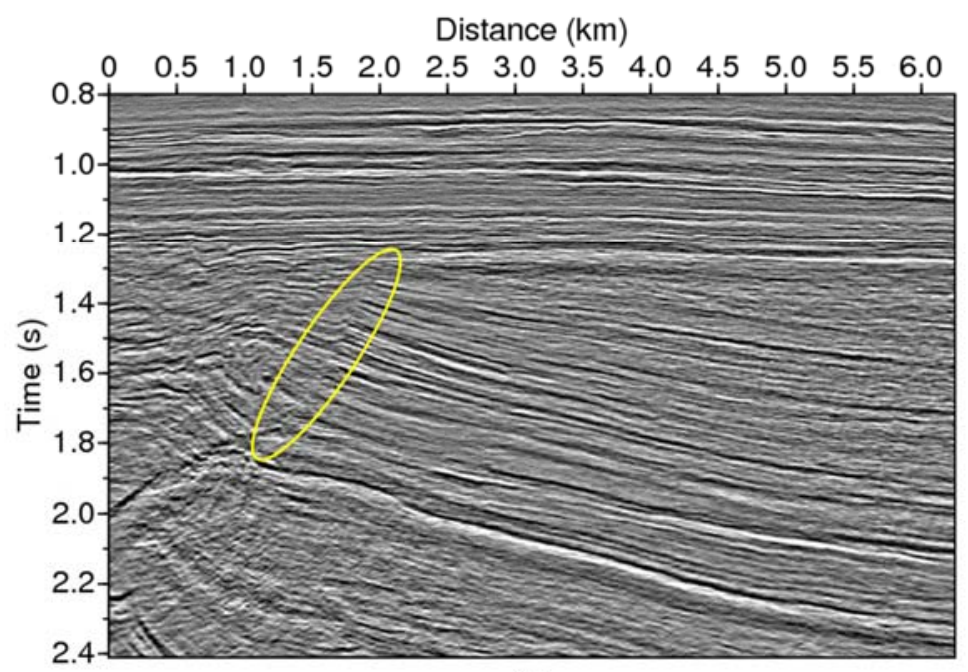

(b)

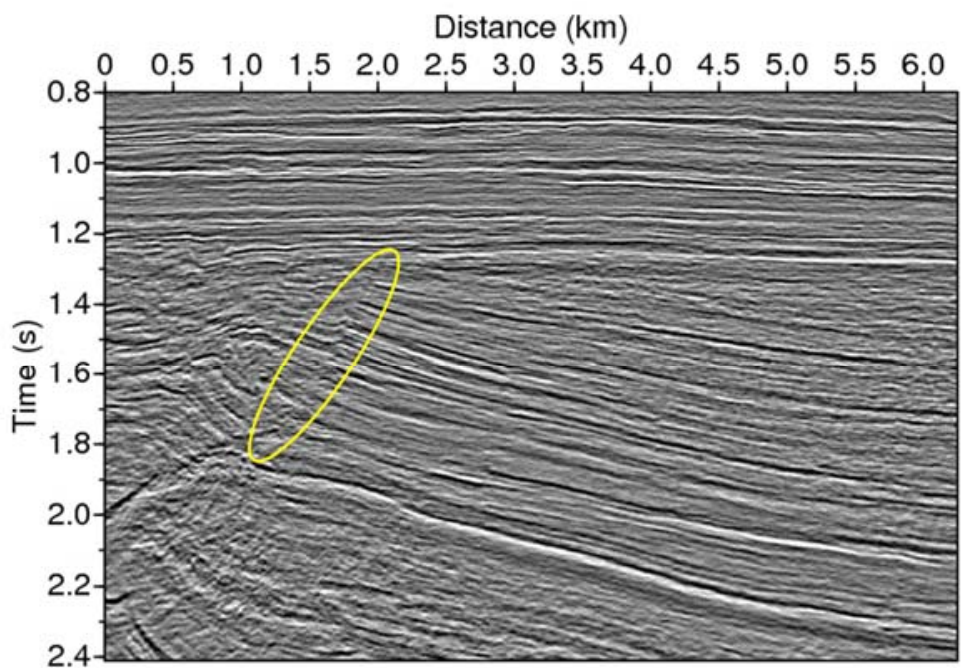

(c)

Figure 11. Gabor deconvolution applied to poststack seismic data. (a) Migration section before Gabor deconvolution. (b) The result of Gabor deconvolution using hyperbolic smoothing. (c) The result of Gabor deconvolution using regularized smoothing. All the figures are plotted in the same scale. 


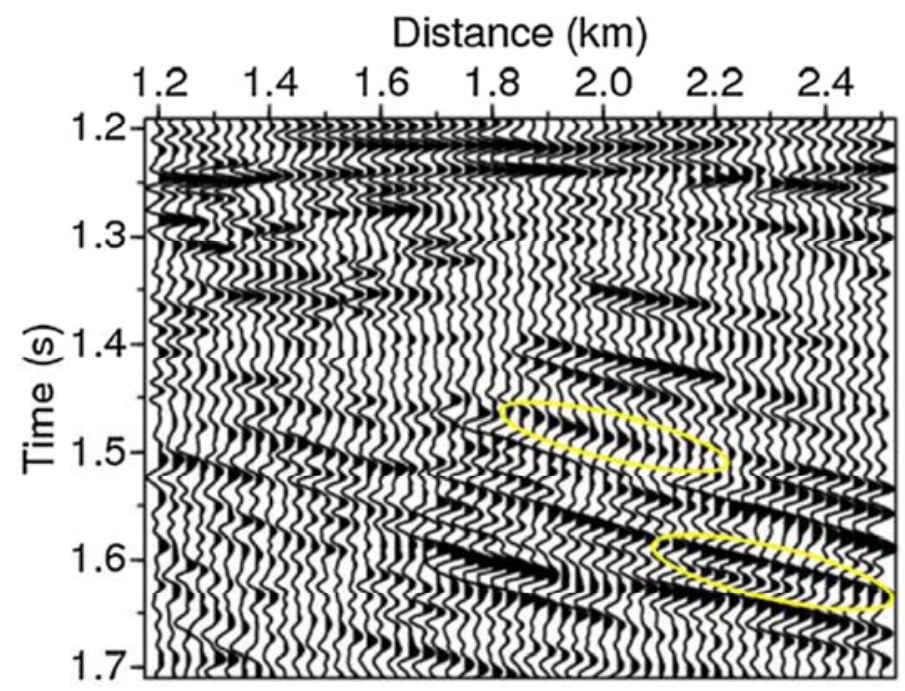

(a)

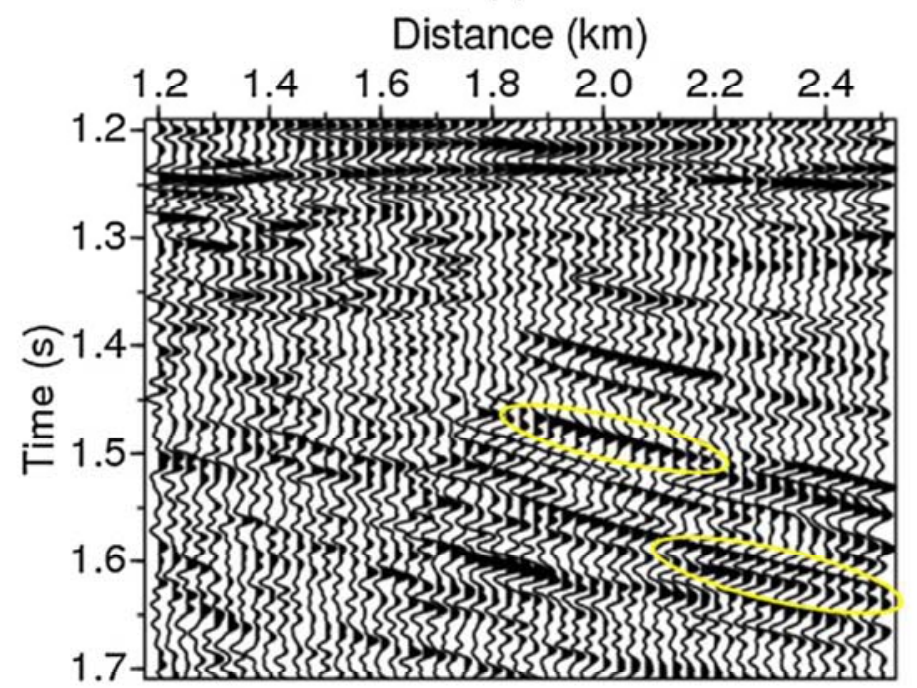

(b)

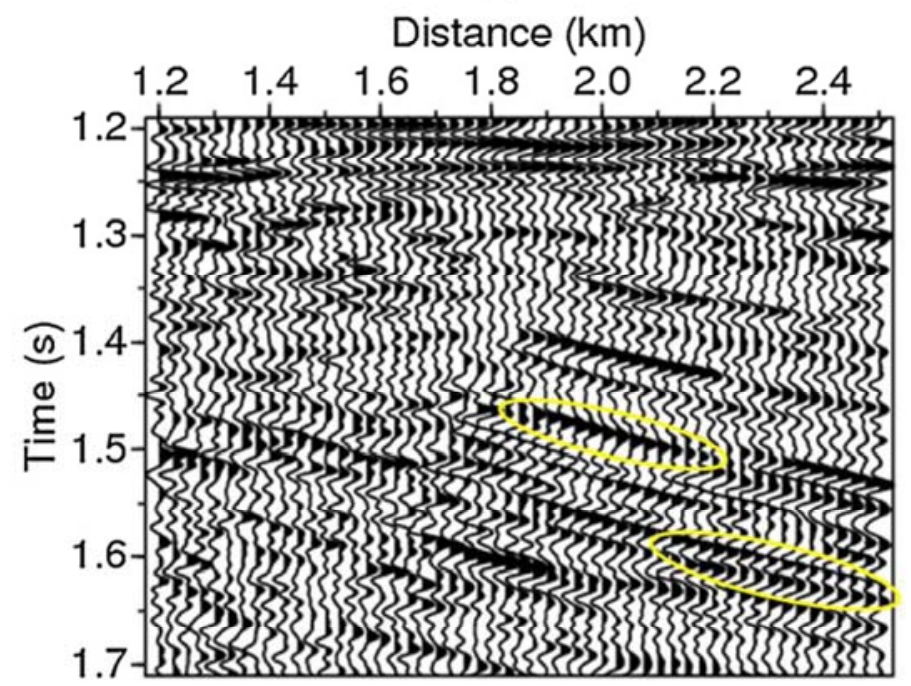

(c)

Figure 12. Zoomed-in sections of the rectangular regions in figure 11. (a) Migration section before Gabor deconvolution. (b) The result of Gabor deconvolution using hyperbolic smoothing. (c) The result of Gabor deconvolution using regularized smoothing. The ovals indicate the areas of comparison. 


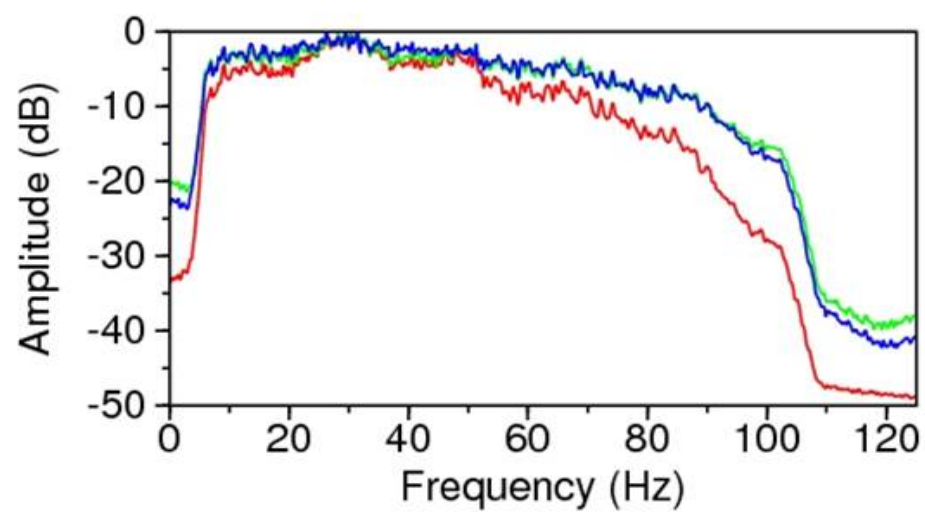

Figure 13. A comparison among the average amplitude spectra of the seismic sections shown in figure 9 before (red) and after applying Gabor deconvolution using hyperbolic smoothing (green) and regularized smoothing (blue).

In figure 13, compared the average amplitude spectra of the seismic sections shown in figure 11, Gabor deconvolution recovers all the frequency components that are in principle recoverable. In this case, the regularized smoothing method can be an alternative of the hyperbolic smoothing method to determine the Gabor deconvolution operator.

It concludes from this field data example that Gabor deconvolution is a promising tool to improve seismic resolution in both prestack and poststack data processing, provided the ambient and coherent noises are carefully eliminated in the preprocessing stage.

\section{Discussion}

The Gabor deconvolution generates a high-resolution reflectivity series, which has a strong correlation with the true reflectivity series. Above all, this has been done without knowing or estimating the Q values (Wang 2004). Compared with the inverse Q filtering, Gabor deconvolution does not suffer from the instability problem (Wang 2002, 2006). It not only eliminates the attenuation effect, but also removes the source wavelet. Therefore, the seismic resolution is enhanced remarkably by the Gabor deconvolution.

When seismic waves travel a certain distance, the amplitude of the high-frequencywave component is attenuated to a level below the ambient noise level (30-70 dB). Gabor deconvolution or stabilized inverse Q filtering (Wang 2006) does not attempt to recover it by using a stabilization factor. In the two synthetic data examples, compared with the true reflectivity (figure 5(a) or figure 7(a)), the Gabor deconvolution results (figures 5(e), ( f ) or figures 7(e), ( f )) display higher resolution in the upper part (0-2 s) than in the deep parts (24 s) due to problems with operator estimation at high attenuations.

The Gabor deconvolution can be applied to prestack and poststack data to improve seismic resolution. The average $\mathrm{Q}$ describes a total apparent effect including intrinsic attenuation and stratigraphic filtering. The Gabor deconvolution operator (equation (13)) takes advantage of the information from a whole seismic trace. Therefore, the Gabor deconvolution can be performed trace by trace and there is no need to deal with the lateral attenuation affect in the prestack gather.

Both Gabor deconvolution and the general wavelet deconvolution assumes that the source wavelet is minimum phased and the reflectivity is statistically white. However, the Gabor deconvolution extends the stationary deconvolution to nonstationarity which is due to attenuation processes. For an attenuated trace, as the wavelet is evolving along the traveltime, 
the general wavelet deconvolution can be applied after inverse Q filtering or by windowing the trace. Nevertheless, it is difficult to estimate the exact Q model from seismic data or the windows should be variable in order to accommodate the time-variant seismic wavelet. The Gabor deconvolution algorithm combines the stationary deconvolution and inverse Q filtering together to eliminate the propagating wavelet. Apparently, the result of Gabor deconvolution is superior to that of a general wavelet deconvolution (Margrave et al 2011, Ahadi and Riahi 2013).

In Gabor deconvolution, minimum-phase assumption about source wavelet and its attenuation process ensure that the phase spectrum of the propagating wavelet can be determined by the smoothed amplitude spectrum. For the real seismic data, it does not always satisfy the minimum-phase assumption due to the noise influence, but the physical attenuation is always minimum phased. We may only estimate the average-Q attenuation by hyperbolic smoothing, and design the attenuation compensation operator instead of Gabor deconvolution operator, then Gabor deconvolution yields to an attenuation compensation method that deals with the minimum-phased attenuation.

Residual nonstationary phase-rotation remaining after Gabor deconvolution is caused by discrete computation of the Hilbert transform (equation 11). This remaining phase-rotation can be corrected by simply resampling the trace into a smaller sample rate if the attenuation is weak (Montana, 2005), or nonstationary phase estimation using regularized local kurtosis maximization (van der Baan and Fomel, 2009) or local similarity with the envelop (Fomel and van der Baan, 2010).

\section{Conclusions}

This paper demonstrates the following two advantageous aspects:

(1)The Gabor deconvolution is a fully data-driven method for reflectivity estimation. It yields a high-resolution estimate of the reflectivity, even for a strong attenuation, but without knowing or estimating the $Q$ values.

(2)Compared with the hyperbolic smoothing method, the regularized smoothing method can be an alternative or even better to determine the Gabor deconvolution operator.

However, it should be applied to field seismic data with a high signal-to-noise ratio either in the prestack or poststack domain.

\section{Appendix A: Gabor transform pair}

For the sake of completeness, we summarize the Gabor transform pair we used in this paper. The forward Gabor transform of a signal $s(t)$ is defined as (Gabor, 1946)

$$
S_{g}(\tau, f)=\int_{-\infty}^{\infty} s(t) h(t-\tau) e^{-2 \pi i f t} d t,
$$

where $h(t)$ is the Gabor analysis window, $\tau$ is the location $\mathrm{f}$ tohe window centre and $f$ is the frequency. The forward Gabor transform can be calculated with a fast Fourier transform (FFT) of the Gabor slices $s(\tau, t)=s(t) h(t-\tau)$, for all possible $\tau$ locations.

The Gabor analysis window at the location $\tau$ is often chosen to be a Gaussian window

$$
h(t-\tau)=\frac{1}{T \sqrt{\pi}} e^{-\frac{(t-\tau)^{2}}{T^{2}}},
$$

where $T$ is referred to as the half width of the Gaussian window. The Gaussian window is symmetric in time and frequency and uniquely minimizes the quadratic time-frequency 
moment about a time-frequency point (Janssen, 1991). Furthermore, there are no side lobes in a Gaussian function which means that a local maximum in the absolute value of the Gabor transform is not an artefact.

In order to reconstruct the time-domain signal $s(t)$ from the forward Gabor transform spectrum $S_{g}(\tau, f)$, the inverse Gabor transform is defined as (Wang, 2006, 2008)

$$
s(t)=\gamma(t) \int_{-\infty}^{\infty} \int_{-\infty}^{\infty} S_{g}(\tau, f) e^{2 \pi i f t} d f d \tau,
$$

where $\gamma(t)$ is the Gabor synthesis window expressed in terms of Gabor analysis window $h(t)$ as

$$
\gamma(t)=\left[\int_{-\infty}^{\infty} h(t-\tau) d \tau\right]^{-1} .
$$

The inner integral in equation (A.3) is an inverse FFT with respect to frequency, which reproduces the Gabor slice. An advantage of this definition is that it can mitigate the potential numerical errors caused by digitization on the Gabor analysis window and the edge effect when moving the analysis window towards both ends of the signal.

As a test of this scheme of Gabor transform, the seismic signal after forward and inverse Gabor transform is compared to the original signal in Figure A.1. The differences, due to discrete implementation and the finite length of Gaussian windows in use, are small enough for inverse $Q$ filtering.
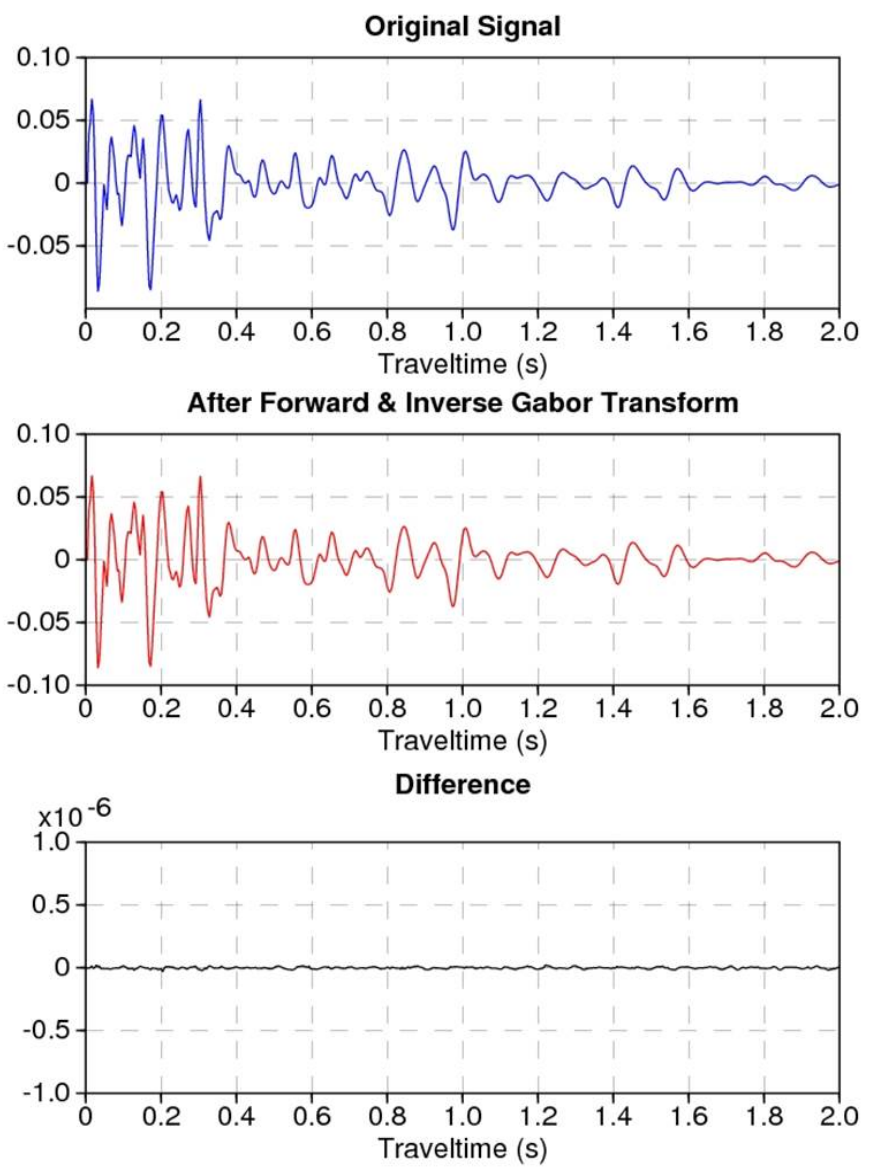

Figure A.1. The result of a forward and inverse Gabor transform (equation A.1 and A.3) is compared with the original signal. The differences are small enough for Gabor deconvolution. 


\section{Acknowledgements}

We are grateful to the sponsors of the Centre for Reservoir Geophysics, Imperial College London, for supporting this research. The first author would like to thank China Scholarship Council for their financial support to study at Imperial College London. This research is also supported by National Science and Technology of Major Projects of China (No. 2011ZX05023-005-005).

\section{References}

Ahadi A. and Riahi M.A. 2013. Application of Gabor deconvolution to zero-offset VSP data. Geophysics 78, D85-D91.

Anderson D.L., Kanamori H., Hart R.S. and Liu H.P. 1977. The Earth as a seismic absorption band. Science 196, 1104-1106.

Bickel S.H. and Natarajan R.R. 1985. Plane-wave $Q$ deconvolution. Geophysics 50, 1426-1439.

Fomel S. 2007. Shaping regularization in geophysical estimation problems. Geophysics 72, R29-R36.

Fomel S. and van der Baan M. 2010. Local similarity with the envelope as a seismic phase detector. 80th Annual International Meeting, SEG, Expanded Abstracts, 1555-1558.

Futterman W.I. 1962. Dispersive body waves. Journal of Geophysical Research 67, 5279-5291.

Gabor D. 1946. Theory of communication. Journal of the Institution of Electrical Engineers 93, 429457.

Hargreaves N.D. and Calvert, A.J. 1991. Inverse $Q$ filtering by Fourier transform. Geophysics 56, 519527.

Iliescu V. and Margrave G.F. 2002. Reflectivity amplitude restoration in Gabor deconvolution. CSEG Annual Convention, Expanded Abstracts.

Janssen A. 1991. Optimality property of the Gaussian window spectrogram. IEEE Transactions on Acoustics, Speech and Signal Processing 39, 202-204.

Kjartansson E. 1979. Constant $Q$ wave propagation and attenuation. Journal of Geophysical Research 84, 4737-4748.

Margrave G.F. 1998. Theory of nonstationary linear filtering in the Fourier domain with application to time-variant filtering. Geophysics 63, 244-259.

Margrave G.F., Dong L., Gibson P., Grossman J.P., Henley D. and Lamoureux M.P. 2003. Gabor deconvolution: Extending Wiener's method to nonstationarity. CREWES Research Report, 15.

Margrave G.F., Henley D.C. and Lamoureux M.P., Iliescu V.F. and Grossman J.P. 2003. Gabor deconvolution revisited. 73th Annual International Meeting, SEG, Expanded Abstracts, 714-717.

Margrave G.F. and Lamoureux M.P. 2002. Gabor deconvolution. CSEG Annual Convention, Expanded Abstracts.

Margrave G.F., Lamoureux M.P. and Henley D.C. 2011. Gabor deconvolution: Estimating reflectivity by nonstationary deconvolution of seismic data. Geophysics 76, W15-W30.

Montana C.A. and Margrave G.F. 2005. Phase correction in Gabor deconvolution. 75th Annual International Meeting, SEG, Expanded Abstracts, 2173-2176.

Robinson E.A. 1967. Predictive decomposition of time series with application to seismic exploration. Geophysics 32, 418-484.

van der Baan M. and Fomel S. 2009. Nonstationary phase estimation using regularized local kurtosis maximization. Geophysics 74, A75-A80.

Wang Y. 2002. A stable and efficient approach of inverse $Q$ filtering. Geophysics 67, 657-663.

Wang Y. 2004. $Q$ analysis on reflection seismic data. Geophysical Research Letters 31, L17606.

Wang Y. and Guo J. 2004. Modified Kolsky model for seismic attenuation and dispersion. Journal of Geophysics and Engineering 1, 187-196.

Wang Y. 2006. Inverse $Q$ filter for seismic resolution enhancement. Geophysics 71, V51-V60.

Wang Y. 2008. Seismic Inverse Q Filtering. Blackwell Publishing, Oxford.

Yilmaz O. 2008. Seismic data analysis. SEG. 